\title{
Dispersion analysis of the meshless local boundary integral equation (LBIE) method for the Helmholtz equation
}

\author{
H. Dogan \& V. Popov \\ Wessex Institute of Technology, Environmental and Fluid Mechanics, UK
}

\begin{abstract}
Numerical solutions of the Helmholtz equation suffer from numerical pollution especially for the case of high wavenumbers. The major component of the numerical pollution is, as has been reported in the literature, the dispersion error which is defined as the phase difference between the numerical and the exact wave. The dispersion error for the meshless methods can be a priori determined at an interior source node assuming that the potential field obeys a harmonic evolution of the numerical wavenumber.

In this work the meshless local boundary integral equation (LBIE) in 2D is investigated with respect to the dispersion effect. Radial basis functions, with second order polynomials and frequency dependent polynomial basis vectors, are used for the interpolation of the potential field. The results have been found to be of comparable accuracy with other meshless approaches.

Keywords: meshless methods, dispersion, local boundary integral equation, radial basis functions, Helmholtz equation.
\end{abstract}

\section{Introduction}

Accurate solution of the Helmholtz equation is of importance for many applications in acoustics such as ultrasonics, microfluidics, aeroacoustics, etc. The simulations involving the high frequency oscillations and large scale industrial setups get cumbersome to solve due to immense computational storage and time requirements.

It is well known that numerical solutions of the Helmholtz equation encounter the pollution affect especially for high wavenumbers. The major component of the numerical pollution is the dispersion error which is defined as the relative 
phase difference between the exact and the numerical wave. For the FEM solutions numerical dispersion and pollution have been extensively studied [1]. Considering the meshless methods for acoustic computations, the dispersion error has been investigated for the element-free Galerkin method (EFGM) [2, 3] and radial point interpolation method (RPIM) [4]. The dispersion error of the meshless methods is significantly reduced in comparison to the classical FEM as shown by reported research [2-4]. An extensive optimization work has been recently carried out for the minimization of the dispersion with respect to the internal parameters of the above-mentioned meshless methods [5].

The meshless local boundary integral equation (LBIE) method has been first introduced for the solution of potential problems by Zhu et al. [6] and developed over the years for many engineering problems such as elastostatics, thermoelasticity, etc. [7]. For acoustic applications, Chen et al. [8] have implemented the LBIE pointing out significant findings when solving for the high wavenumbers.

In this paper, the LBIE, using the radial basis functions (RBFs) for the interpolation of the potential field, has been investigated in terms of dispersion error. In Section 2, the RBF formulation of the LBIE is outlined. Dispersion error is stated in Section 3 and some numerical results are shown in Section 4 followed by conclusions in Section 5 .

\section{Local boundary integral equation with radial basis functions}

In this section the local boundary integral equation, using the radial basis functions for the interpolation of the domain variables, for the solution of the Helmholtz equation is introduced. The method will be called as LBIE(RB) throughout the paper for the sake of simplicity, and its differences from the original LBIE will be discussed in this section.

\subsection{Derivation of the governing equations}

Let us consider the following Helmholtz equation:

$$
\left(\nabla^{2}+k^{2}\right) u(r)=0
$$

where $u(r)$ is a potential field, $r$ is the position vector and $k$ is the wavenumber. Given a point $r$ inside a domain $\Omega$, by applying the Green integral formula, the integral representation of (1) can be written as:

$$
\lambda(r) u(r)+\int_{\Gamma} \frac{\partial u^{*}}{\partial n}(r, \xi) u(\xi) d \Gamma_{\xi}-\int_{\Gamma} u^{*}(r, \xi) \frac{\partial u}{\partial n}(\xi) d \Gamma_{\xi}=0
$$

where $r$ and $\xi$ are the source and field points, respectively, $u^{*}(r, \xi)$ is the fundamental solution of the Helmholtz equation and $n$ is the unit outward normal over the boundary, $\Gamma$, of the domain, $\Omega$. For $2 \mathrm{D}$ problems the fundamental solution is given by 


$$
\begin{gathered}
u^{*}(\mathrm{r}, \xi)=-\frac{i}{4} H_{0}^{(2)}(k R) \\
H_{0}^{(2)}(k R)=J_{0}(k R)-i Y_{0}(k R)
\end{gathered}
$$

where $H_{n}^{(2)}$ is the Hankel function of the second kind $n^{\text {th }}$ order, $J_{0}$ and $Y_{0}$ are Bessel functions of the first and second kind, respectively and $R$ is the distance from the source point to the field point under consideration, i.e. $R=|r-\xi|$. The constant $\lambda(r)$ has a value between 0 and 1 being equal to $1 / 2$ for smooth boundaries and 1 if the source point $r$ is inside the domain.

In the LBIE method, equation (2) is written over the local sub-domains $\Omega_{s}$ and the test function is modified with the use of the "companion solution" in order to eliminate the single layer integral. The companion solution is required to be the solution of the following Dirichlet problem,

$$
\begin{gathered}
\nabla^{2} u^{\prime}(\mathrm{r})+k^{2} u^{\prime}(r)=0 \quad \text { on } \Omega_{s}, \\
u^{\prime}(\mathrm{r})=u^{*}(r) \quad \text { on } \partial \Omega_{s} .
\end{gathered}
$$

However, as noted in the literature [7], fundamental solution of (5) is not available. Instead, the companion solution of the Laplace operator will be used:

$$
\begin{aligned}
& \nabla^{2} u^{\prime}(\mathrm{r})=0 \text { on } \Omega_{s}, \\
& u^{\prime}(\mathrm{r})=u^{*}(r) \text { on } \partial \Omega_{s} .
\end{aligned}
$$

which can be used to measure the dispersion error.

By using $u^{* *}=u^{*}-u^{\prime}$ as the test function and by applying the Green integral formula over the local sub-domain $\Omega_{s}$, the integral representation of (1) can be rewritten as:

$$
\begin{aligned}
& \lambda(r) u(r)+\int_{\Omega_{s}} k^{2} u^{\prime}(r, \zeta) u(\zeta) d \Omega_{\zeta}+ \\
& \int_{\partial \Omega_{s}} \frac{\partial u^{* *}}{\partial n}(r, \xi) u(\xi) d \Gamma-\int_{\partial \Omega_{s}} u^{* *}(r, \xi) \frac{\partial u}{\partial n}(\xi) d \Gamma=0
\end{aligned}
$$

where $\zeta$ is the field variable over $\Omega_{s}$. As can be noted, with the aid of (6), the last term in (7) vanishes when the local sub-domain $\Omega_{s}$ is located entirely within the global domain of the problem, $\Omega$. This yields an equation purely in terms of the potential field. When the local sub-domain $\Omega_{s}$, is close to the global boundary of the problem so that its boundary intersects with the global boundary, the single layer integral must be evaluated. This could be done by integration over the global boundary [6] or by applying some collocation 
techniques [9]. Nevertheless, as detailed in Section 3, dispersion error is measured for the potential field at the source nodes which are located entirely within the global domain and not affected by the acoustic forcing terms or any type of boundary conditions.

Equation (7) requires the integration of the function $u^{\prime}(r, \zeta)$ over the local sub-domains. Although local sub-domains can be of any shape, circular subdomains are used in this work, therefore the companion solution is simply obtained as,

$$
u^{\prime}(r, \zeta)=-\frac{i}{4} H_{0}^{(2)}\left(k R_{\Omega_{s}}\right)
$$

where $R_{\Omega_{s}}$ is the radius of the sub-domain. Note that, using Laplace fundamental solution when applying the Green integral formula to (1) would also result in evaluation of domain integrals for the potential field.

\subsection{Interpolation of the field variable using radial basis functions}

In order to perform the integration over the local sub-domains and the boundaries of the local sub-domains, values of the potential field must be known. For the integration over the boundary, a simple Gaussian integration procedure can be applied over the polar angle as shown by Ooi and Popov [10], without the necessity of the discretization of the local boundary. As for the domain integrals, Gaussian integration must be performed both over the radial direction and the angular direction. The unknown potential at one of these integration points is approximated by using radial basis functions (RBFs). In order to ensure the stability of the approximation, polynomial terms are inserted into the basis $[4,5]$. The potential at any location $\vartheta$ is approximated by $n$ neighbouring nodes (see Figure 1):

$$
u(\vartheta)=\sum_{i=1}^{n} f\left(\vartheta, x_{i}\right) \cdot a_{i}+\sum_{j=1}^{m} p\left(\vartheta, x_{j}\right) \cdot b_{j}
$$

where $f$ is the RBF used, $p$ is the polynomial basis with $m$ terms, and $a_{i}, b_{j}$ are unknown coefficients. The second order augmented thin plate spline $f(R)=R^{4} \ln (R)$ is used throughout this paper. The selection of the $n$ neighbouring nodes is done by defining a square shaped influence domain with varied radii as explained in Section 3.

The choice of the polynomial basis vector affects the solution significantly. As reported in the literature $[4,5]$, polynomials with $m=6$ can used.

$$
\vec{p}(x, y)=\left[\begin{array}{llllll}
1 & x & y & x y & x^{2} & y^{2}
\end{array}\right]
$$




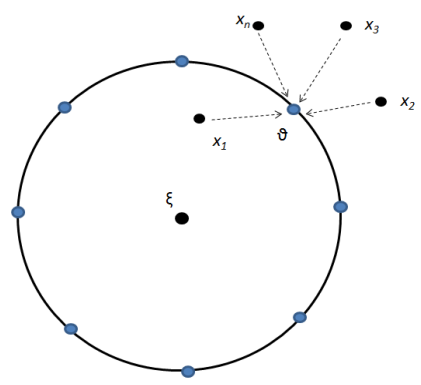

Figure 1: Interpolation of the values at the integration points.

The use of frequency dependent polynomial basis vectors has been shown to produce accurate approximations for the Helmholtz problems:

$$
\begin{array}{rcc}
\vec{p}(x, y)=\left[\begin{array}{llc}
1 & \cos (k x \cos \beta+k y \sin \beta) & \sin (k x \cos \beta+k y \sin \beta) \\
& \cos (-k x \sin \beta+k y \cos \beta) & \sin (-k x \sin \beta+k y \cos \beta)
\end{array}\right]
\end{array}
$$

with $m=5$. Here, $k$ denotes the wavenumber and $\beta$ the angle of propagation for which the solution is assumed to be exact. In this work, two different propagation angles are examined for the dispersion error of the method, namely $\beta=0$ and $\beta=\pi / 4$.

Equation (9) can be written in matrix form as:

$$
\mathbf{U}_{0}=\boldsymbol{F}_{0} \boldsymbol{a}+\boldsymbol{P}_{\mathbf{0}} \mathbf{b}
$$

Moreover, in order to have a unique approximation of a function,

$$
\boldsymbol{P}_{0}^{T} \mathbf{a}=0
$$

has to be fulfilled. Since $\boldsymbol{F}_{0}$ is convertible equation (12) can be transformed as:

$$
\boldsymbol{a}=\boldsymbol{F}_{0}^{-1} \mathbf{U}_{0}-\boldsymbol{F}_{0}^{-1} \boldsymbol{P}_{0} \mathbf{b}
$$

Using (13), equation (12) yields

$$
\boldsymbol{b}=\boldsymbol{R}_{\boldsymbol{b}} \mathbf{U}_{0}
$$

with

$$
\boldsymbol{R}_{\boldsymbol{b}}=\left[\mathbf{P}_{0}^{T} \mathbf{F}_{0}^{-1} \boldsymbol{P}_{\boldsymbol{0}}\right]^{-1} \mathbf{P}_{0}^{T} \mathbf{F}_{0}^{-1}
$$

Using the expression (15) for $\boldsymbol{b}$ in (14) yields:

$$
\boldsymbol{a}=\boldsymbol{R}_{\boldsymbol{a}} \mathbf{U}_{0}
$$

where

$$
\boldsymbol{R}_{a}=\mathbf{F}_{0}^{-1}\left[1-\boldsymbol{P}_{0} \boldsymbol{R}_{b}\right]
$$


Finally, substitution of (15) and (18) into the equation (9) the potential at the point $\vartheta$ can be obtained:

$$
U(\vartheta)=F\left(\vartheta, x_{i}\right) \mathbf{R}_{a} u_{0}+P\left(\vartheta, x_{j}\right) \mathbf{R}_{b} u_{0}
$$

where $F\left(\vartheta, x_{i}\right)=\left[f\left(\vartheta, x_{1}\right), f\left(\vartheta, x_{2}\right), \ldots, f\left(\vartheta, x_{n}\right)\right]$ and $P\left(\vartheta, x_{j}\right)=\left[p\left(\vartheta, x_{1}\right)\right.$, $\left.p\left(\vartheta, x_{2}\right), \ldots, p\left(\vartheta, x_{m}\right)\right]$.

\section{Dispersion effect}

Solution of the Helmholtz equation suffers the so called pollution effect, which leads to inaccurate results especially for the case of high wave numbers. It has been mathematically shown that the pollution mainly consists of the dispersion effect which is defined as the mismatch between the actual wavenumber, $k$, and the numerical one, $k^{h}$. For the FEM solutions of the Helmholtz equation an error estimate is given by [1],

$$
e_{h}=C_{1} k h+C_{2} k^{3} h^{2}, \quad k h<1
$$

with $h$ is the distance between nodal points in an assumed uniform setting of the nodes and the constants $C_{1}$ and $C_{2}$ independent of $k$ and $h$. The first term in (20) is related as the approximation error and the second as the dispersion error.

Given a regular distribution of field points and with a constant size of influence domains, the numerical wavenumber $k^{h}$ of the numerical wave propagating in the direction of $\beta$ can be a priori determined as mathematically outlined in detail by Suleau et al. [3] which will be repeated here for the LBIE(RB) method.

It is assumed that the nodal values of the potential field follow a harmonic evolution of the form

$$
u^{h}\left(x_{q_{1}}, y_{q_{2}}\right)=A \exp \left(j k^{h}\left(x_{q_{1}} \cos \beta+y_{q_{2}} \sin \beta\right)\right)
$$

and after the RBF interpolation, the potential at an interior node $\left(x_{q_{1}+\gamma_{1}}, y_{q_{2}+\gamma_{2}}\right)$ can be written as:

$$
u^{h}\left(x_{q_{1}+\gamma_{1}}, y_{q_{2}+\gamma_{2}}\right)=u^{h}\left(x_{q_{1}}, y_{q_{2}}\right) \exp \left(j k^{h}\left(\gamma_{1} h \cos \beta+\gamma_{2} h \sin \beta\right)\right)
$$

In the $\operatorname{LBIE}(\mathrm{RB})$, given a constant size of influence domain, a discrete equation is written for the interior nodes of the form:

$$
\sum_{I=-s_{1}}^{+s_{1}} \sum_{J=-s_{2}}^{+s_{2}} X_{I, J} u^{h}\left(x_{q_{1}+I}, y_{q_{2}+J}\right)=0
$$

with $s_{1}, s_{2}$ being the normalized (with $h$ ) distances of the furthest neighbouring nodal points which remain within the influence domain of $\left(q_{1}, q_{2}\right)$ in $x$ and $y$ directions, respectively. Here, $X_{I, J}$ is the coefficient of the potential value $u^{h}\left(x_{q_{1}+I}, y_{q_{2}+J}\right)$ arising in the discretized form of the equation (7) written for the node at $\left(q_{1}, q_{2}\right)$. 
By assuming the harmonic evolution of the acoustic pressure of the nodal values given by (22), equation (23) becomes:

$$
\sum_{I=-s_{1}}^{+s_{1}} \sum_{J=-s_{2}}^{+s_{2}} X_{I, J} u^{h}\left(x_{q_{1}}, y_{q_{2}}\right) \exp \left(j k^{h}(I h \cos \beta+J h \sin \beta)\right)=0 .
$$

For a non-zero distribution of the acoustic pressure, $u^{h}$, it obvious from the equation (24) that the numerical wavenumber can be determined, for given values of $k$ and the angle of propagation $\beta$, by solving:

$$
\sum_{I=-s_{1}}^{+s_{1}} \sum_{J=-s_{2}}^{+s_{2}} X_{I, J} \exp \left(j k^{h}(I h \cos \beta+J h \sin \beta)\right)=0 .
$$

Dispersion error: Throughout this paper we present the results for dispersion as follows:

$$
\varepsilon=\frac{k h-k^{h} h}{k h}
$$

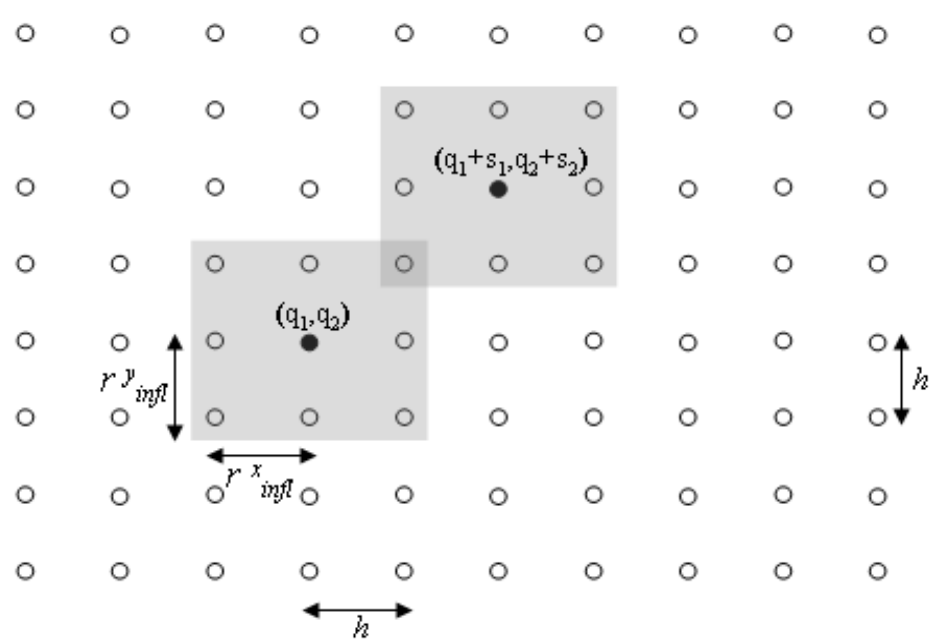

Figure 2: Influence domains of two associated points located at $\left(\mathrm{q}_{1}, \mathrm{q}_{2}\right)$ and $\left(\mathrm{q}_{1}+\mathrm{s}_{1}, \mathrm{q}_{2}+\mathrm{s}_{2}\right)$.

\section{Numerical results}

The dispersion error for the solution of the Helmholtz equation with LBIE(RB) is investigated in this section. It depends mainly on the choice of the polynomial and the number of nodes used in the RBF interpolation. In this study square influence domains are considered with varying radius of 
influence $r_{\text {inf } l}=h, 2 h, 3 h, 4 h$. Three different polynomial basis vectors are used as mentioned through equations (10)-(11). The basis shown in (10) will be referred to as $\mathrm{m}=6$, while the basis given in (11) will be referred to as $\beta=0$ and $\beta=\pi / 4$.

Figures 3 and 4 show the dispersion for the $\operatorname{LBIE}(\mathrm{RB})$ with $\mathrm{m}=6$ for the propagation angle $\beta=0$. It can be noticed in Figure 3 that dispersion increases with increasing wavenumber. This is fact which makes it difficult for the solution of high wavenumber problems for almost all of the numerical methods. Also notice that increasing the $r_{\text {infl }}$ leads to improved results.

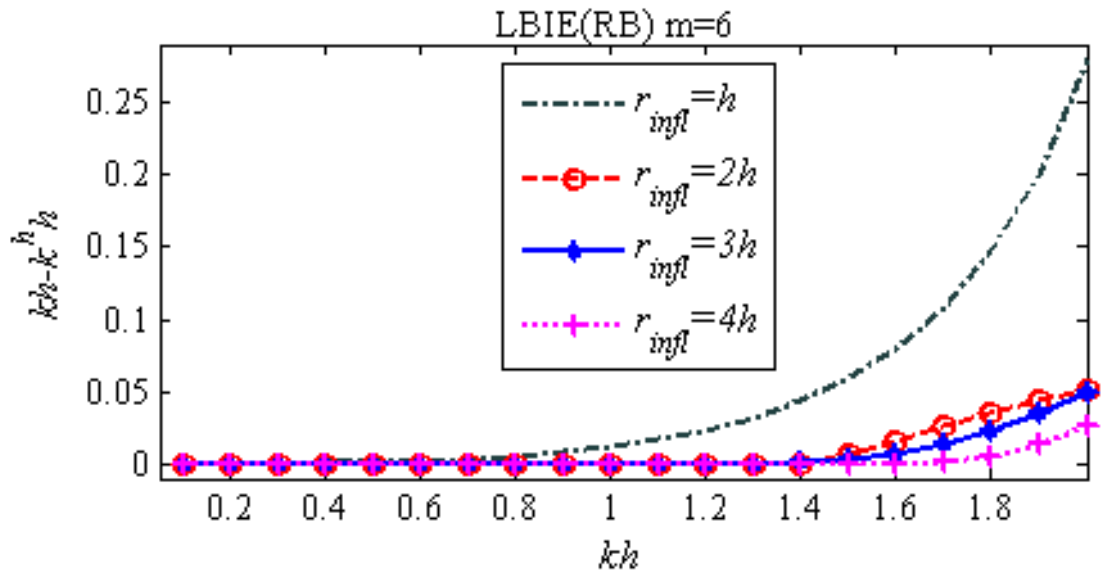

Figure 3: Difference between the non-dimensionalized exact and numerical wavenumber.

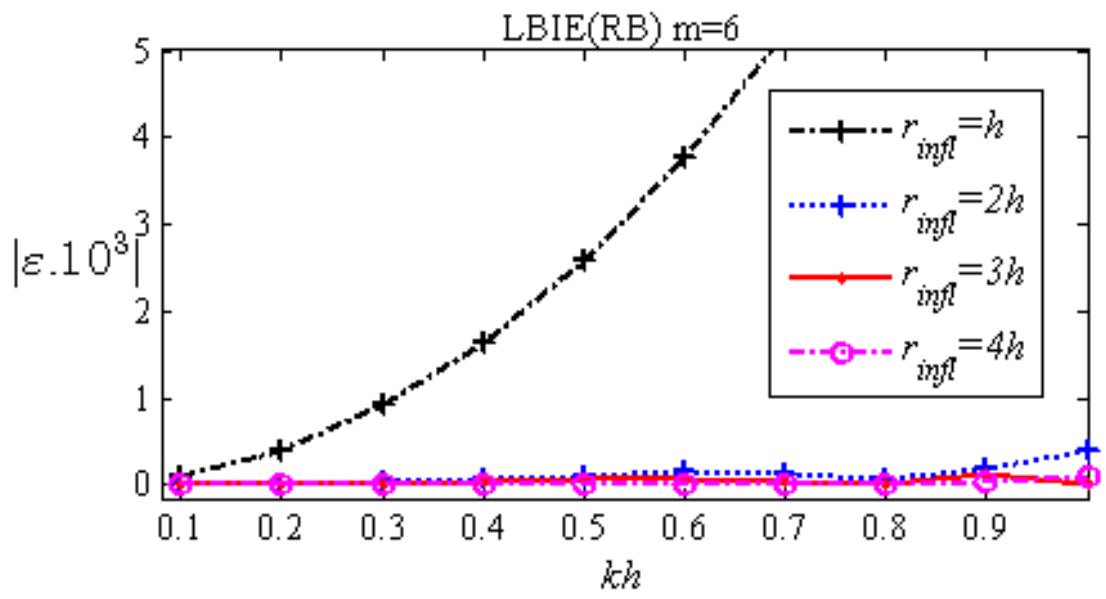

Figure 4: Dispersion error over the non-dimensionalized exact wavenumber for the propagation angle $\alpha=0$. 
Figure 5 shows the dispersion error over the propagation angle $\alpha$ for two different values of $k h, 1$ and 2 respectively. For the $\operatorname{LBIE}(\mathrm{RB})$ with $\beta=0$ and LBIE(RB) with $\beta=\pi / 4$, the methods are regarded as dispersion-free when the angle of propagation coincides with the angle of the polynomial basis vector.
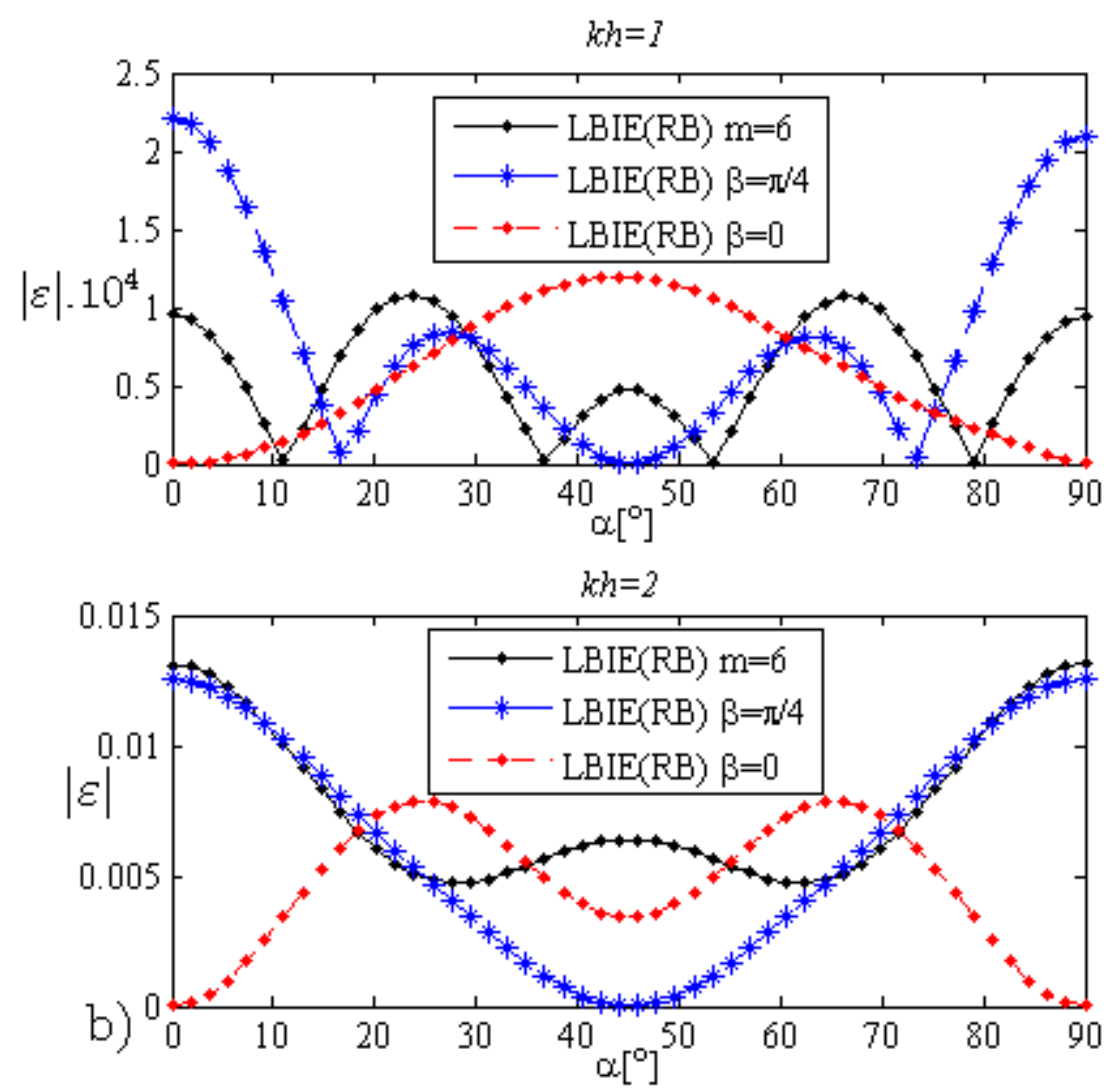

Figure 5: Dispersion error over the propagation angle $\beta$ : (a) $k h=1$ and (b) $k h=2$.

In Figure 6, an overall measure of dispersion is shown for the varying range of $r_{\text {inf } l}=2 h, 3 h, 4 h, \beta=[0, \pi / 2]$ and $k h=[0,3]$. Note that increasing the $r_{\text {infl }}$ leads to more accurate results being consistent with the previous results. It can be observed that the results get distorted when $k h>1.5$ which also increases the pollution effect significantly rendering the methods impractical. For the range $k h<1.5$, further investigations should be carried out in order to compare the performances of the different approaches. 

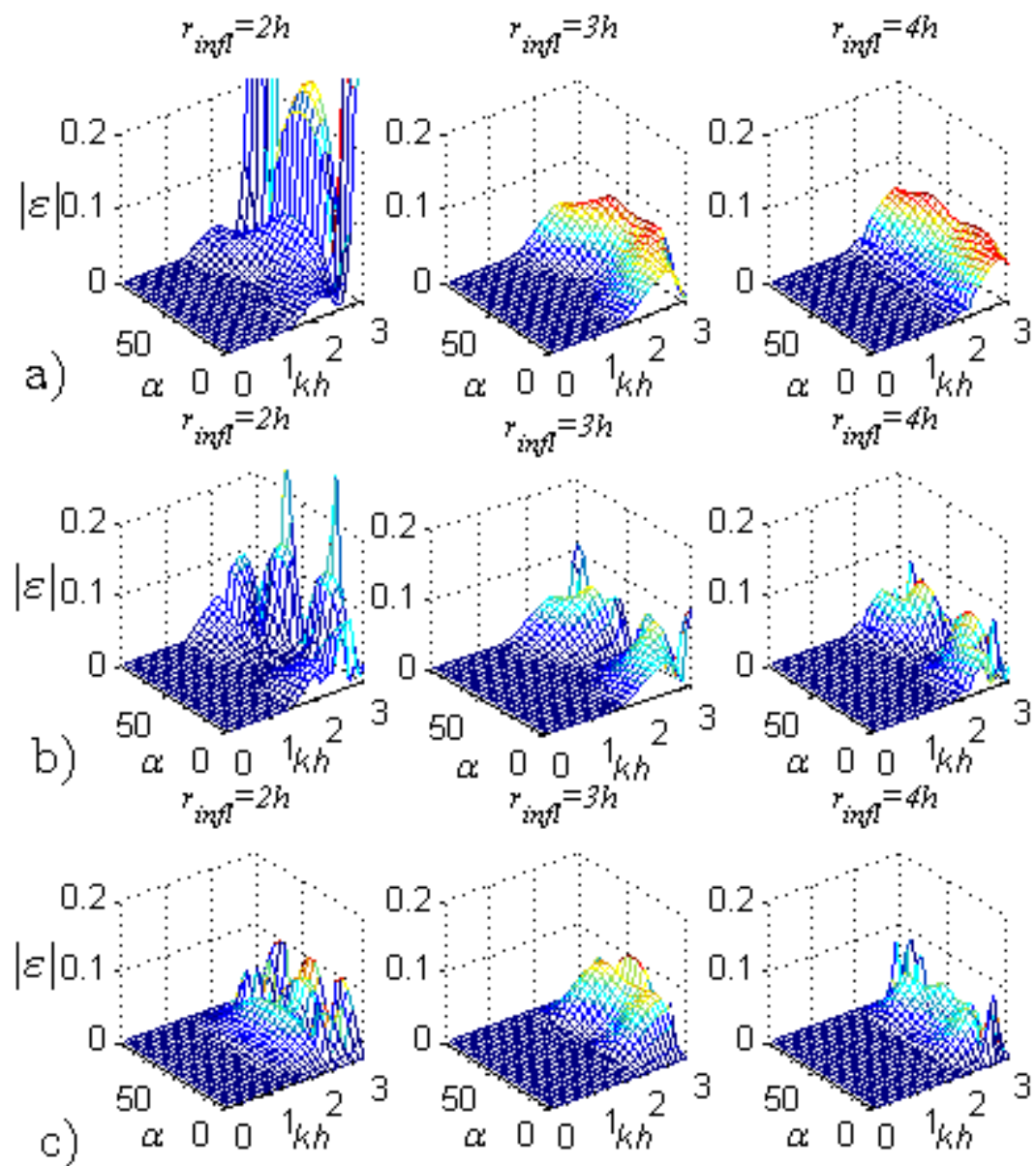

Figure 6: Dispersion error over $\alpha$ and $k h$ : (a) $\operatorname{LBIE}(\mathrm{RB}) \mathrm{m}=6$, (b) $\operatorname{LBIE}(\mathrm{RB}) \beta=\pi / 4$ and (c) $\operatorname{LBIE}(\mathrm{RB}) \beta=0$.

\section{Conclusions}

The dispersion error when solving the Helmholtz equation with local boundary integral equation (LBIE) has been investigated. Radial basis functions, with second order polynomials and frequency dependent polynomial basis vectors were used for the interpolation of potential field. It has been shown previously in the literature that, meshless methods have less dispersion error compared to classical FEM [2-5]. The results presented are found to be in the same order of accuracy with other meshless approaches such as radial point interpolation method [3] and element-free Galerkin method [2], though a direct comparison of 
the methods has not been carried out. The results presented here, as giving a better understanding of how to reduce the pollution error for the Helmholtz equation, are of importance for meshless methods applied to acoustic problems in the high frequency range.

\section{Acknowledgement}

The present study was supported by the SONO project, contract number: 228730, as part of the Seventh Framework Programme (FP7-NMP-2008-Large2).

\section{References}

[1] Deramaeker A., Babuska I. and Bouillard P. Dispersion and pollution of the FEM solution for the Helmholtz equation in one, two and three dimensions. Int. J. Numer. Methods Eng. 1999; 46: 471-499.

[2] Bouillard $\mathrm{Ph}$. and Suleau S. Element-Free Galerkin solutions for Helmholtz problems: formulation and numerical assessment of the pollution effect. Comp. Methods Appl. Mech. Eng. 1998; 162: 317-335.

[3] Suleau S., Deramaeker A. and Bouillard P. Dispersion and pollution of meshless solutions for the Helmholtz equation. Comput. Methods Appl. Mech Engrg. 2000; 190: 639-657.

[4] Wenterodt C. and von Estorff O. Dispersion analysis of the meshfree radial point interpolation method for the Helmholtz equation. Int. J. Numer. Methods Eng. 2009; 77: 1670-1689.

[5] Wenterodt C. and von Estorff O. Optimized meshfree methods for acoustics. Comput. Methods Appl. Mech Engrg. 2011; 200: 2223-2236.

[6] Zhu T., Zhang J. D., Atluri S. N. A local boundary integral equation (LBIE) method in computational mechanics, and a meshless discretization approach. Computational Mechanics 1988; 21: 223-235.

[7] Sladek J., Sladek V., Atluri S. N. Application of the local boundary integral equation method to boundary-value problems. Int. Applied Mechanics 2002; Vol. 38(9): 1025-1047.

[8] Chen H. B., Fu D. J. and Zhang P. Q. An investigation of wave propagation with high wavenumbers via the regularized LBIEM. CMES 2007; 20: 8598.

[9] Nicomedes W. L., Mesquita R. C. and Moreira F. J. S. A meshless local Petrov-Galerkin method for three-dimensional scalar problems. IEEE Transactions on Magnetics. 2011; 47: 1214-1217.

[10] Ooi E. H. and Popov V. An efficient implementation of the radial basis integral equation method. Engineering Analysis with Boundary Elements 2012; 36: 716-726. 